(Kupfer et al, 1988). Moreover, bipolar I patients might be more likely to receive concurrent antimanic treatment because of a greater concern about treatment-emergent mania. The proportion of bipolar subtypes could bias the rate of treatmentemergent mania.

Finally, the rate of mania increases from the placebo to the TCA to the SSRI unipolar groups. The rates are significantly greater for drug groups compared with placebo, but the significance between the antidepressant groups is not reported. This trend hints at a possible heightened sensitivity to SSRIs. Fluoxetine may be more effective than imipramine in bipolar depression (Benfield et al, 1986; Cohn et al, 1989), further suggesting that bipolar patients are more sensitive to SSRIs and have a greater risk for treatment-emergent mania.

The problems associated with TCAs have been recognised only after many decades of clinical use. SSRIs are believed to be a 'safer' class of antidepressants, but there has not been sufficient clinical experience to support this notion. Claims about the superior safety of the SSRIs must therefore be carefully and critically evaluated. Hypomanic symptoms are notoriously under-recognised, but are often a prodrome to bipolar disorder (Howland \& Thase, 1993). Because patients are more likely to seek treatment for depression, a careful assessment of risk factors for bipolar disorder is imperative before starting an antidepressant. The SSRIs now enjoy widespread use, especially by non-psychiatric physicians, and many depressed patients seek treatment in non-psychiatric settings (Howland, 1993). Hence, many depressed patients may not have been adequately evaluated and may be at risk for antidepressant-induced mania.

Benfield, P., HeEl, R. C. \& Lewis, S. P. (1986) Fluoxetine. Drugs, 32, 481-508.

Cohn, J. B., Colluns, G., Ashrrook, E., et al (1989) A comparison of fluoxetine imipramine and placebo in patients with bipolar depressive disorder. International Clinical Psychopharmacology, 4, 313-322.

HowlaND, R. H. (1993) Health status, health care utilization, and medical comorbidity in dysthymia. International Journal of Psychiatry in Medicine, 23, 211-238.

— \& Thase, M. E. (1993) A comprehensive review of cyclothymic disorder. Journal of Nervous and Mental Disease, 181, 485-493.

Kupfer, D. J., Carpenter, L. L. \& Frank, E. (1988) Possible role of antidepressants in precipitating mania and hypomania in recurrent depression. American Journal of Psychiatry, 145, 804 808.

Department of Psychiatry

ROBERT H. HOWLAND

University of Pittsburgh School of Medicine

3811 O'Hara Street

Pittsburgh, PA 15213

\section{Substance abuse in schizophrenics}

SIR: Smith \& Hucker (BJP, July 1994, 165, 13-21) reviewed the literature on substance abuse in schizophrenics, focusing on the link between substance abuse and violence, and showed substance abuse as a possible "marker of either some underlying propensity or of an increased likelihood of aggression under certain conditions". Having addressed the problem of delinquency and violent behaviour in schizophrenics recently (Soyka, 1993), their conclusions require discussion.

High rates of substance abuse and dependence have been reported in North American and in some European samples. For example, in two large German samples of schizophrenic in-patients, lifetime prevalence estimates for substance abuse were found to be $21.8 \%$ and $42.9 \%$, with 3-month prevalence rates being $21.3 \%$ and $29.0 \%$ (Soyka et al, 1993). Whether these high rates reflect a real increase of substance abuse in schizophrenia or not is a matter of controversy. Cuffel (1992) pointed out that recent studies show higher prevalence estimates for substance abuse than older studies, but a variety of reasons may account for the observed differences (Alterman, 1992).

Dual diagnosis schizophrenics are not only at increased risk for violence and aggression but delinquency in general. In the two German samples mentioned above, $25 \%$ of all schizophrenics had been convicted before, mostly for offences against property $(19.5 \%)$ and traffic offences $(4.3 \%)$, while violent behaviour was comparatively rare $(1.8 \%)$. Patients with substance abuse had been convicted more often than other schizophrenics $(40.1 \% \mathrm{v}$. $13.7 \%, P<0.01)$.

Variables that may acount for the apparently higher risk for violent behaviour in dual diagnosis schizophrenics include the psychopathological and social factors reviewed by Hucker \& Smith. Since causes of violence in schizophrenics are multifactorial, in most cases it may be more appropriate to speak of "risk factors" instead of "markers", with substance abuse being one among many. The role of intoxication in the development of violence seems to be overestimated. Boeker \& Haefner (1973) found that only $10.4 \%$ of violent schizophrenics studied were intoxicated at the time of their delinquent action. Further research is needed on the relationship between substance abuse and schizophrenia, especially on the aetiology and treatment of substance abuse.

\footnotetext{
Alterman, A. I. (1992) Methodological issues and prevalence estimates of substance abuse in schizophrenia. Commentary.
} Journal of Nervous and Mental Diseases, 180, 593-594. 
BOEKBR, W. \& HAEFNER, H. (1973) Gewalttaten Geistesgestoerter. Eine psychiatrische-epidemiologische Untersuchung in der Bundesrepublik Deutschland. Berlin: Springer.

Curfel, B. J. (1992) Prevalence estimates of substance abuse in schizophrenia and their correlates. Journal of Nervous and Mental Diseases, 180, 589-593.

SoYkA, M. (1993) Substance abuse and dependency as a risk factor for delinquency and violent behavior in schizophrenic patients how strong is the evidence? Journal of Clinical Forensic Medicine, 1, 3-7

-, Albus, M., Fnnelli, A., et al (1993) Prevalence of alcohol and drug abuse in schizophrenic inpatients. European Archives of Psychiatry and Clincial Neuroscience, 242, 362-372.

Psychiatric Hospital

University of Munich

Nussbaumstrasse 7

80336 Munich, Germany

\section{Psychopathology and 'ecstasy'}

SIR: Maguire et al (BJP, September 1994, 165, 391-395) have presented an interesting, if small, study comparing psychiatric symptoms in patients who have taken MDMA ('ecstasy') with a control group who showed no evidence of drug use. However, having presented useful data they draw conclusions that cannot be sustained by their evidence. The authors appear to believe that the symptoms displayed by the 13 patients had a causal link with the use of MDMA. They report that the symptoms displayed by the eight psychotic patients did not differ from the matched controls. Four of the eight psychotic patients had discontinued MDMA use four or more weeks prior to psychiatric presentation, and one patient had discontinued 26 weeks earlier. There was no clear relationship between MDMA use and the onset of symptoms. Little information is offered as to the course that the patients' illnesses took, other than in case 8 where it would appear that symptoms continued irrespective of MDMA use.

We suggest that, from the available evidence, it is equally likely that these patients were suffering from one of the major psychoses. While it is conceivable that MDMA use precipitated or coloured the symptoms, a firm causal link is not sustainable. Use of drugs of abuse, including MDMA, has become widespread in the general population and the coincidence of a psychotic breakdown with drug-taking is bound to happen frequently. It would be dangerous to draw the conclusion that these disorders should necessarily be classified or managed any differently to other psychotic disorders.

The Clunis Report (Ritchie et al, 1994) describes in detail a case where a history of supposed drug use was uncritically taken as an explanation for the persistently abnormal mental state of a man suffering from schizophrenia. This contributed, albeit in a minor way, to the inadequate management of a man who went on to kill. The case illustrates the potential dangers of mistaken attribution of psychotic states to drug use. It is important that the published literature should not exaggerate the strength of the evidence for putative causal relationships to the exclusion of other plausible explanations.

Rrrchin, J., Dick, D. \& Linghum, R. (1994) The Report of the
Enquiry into the Care and Treatment of Christopher Clunis. London: HMSO.

R. G. POOLE

C. J. BRABBINS

Windsor Mental Health Resource Centre Liverpool L8 $7 L F$

\section{Cognitive therapy}

SIR: We would like to bring to your attention a significant omission from Scott's otherwise excellent article on cognitive-behavioural therapy or CBT (BJP, July 1994, 165, 126-130). While she does justice to its application to mood and anxiety disorders, she makes no mention of its application to eating disorders and bulimia nervosa in particular. This omission is surprising since the evidence supporting this specific application of CBT is at least as strong as in other areas, if not more so.

The rationale for using a cognitively-oriented treatment for bulimia nervosa arises from the prominence of these patients' extreme concerns about shape and weight and their likely role in the maintenance of the disorder (Wilson \& Fairburn, 1993). This application of CBT has been the subject of over 15 randomised controlled trials and their findings support its use (Fairburn et al, 1992). Once maintenance of change is taken into account, it is clear that the effects of CBT are superior to those of all other treatments studied, with the possible exception of interpersonal psychotherapy (Fairburn et al, 1993a). Between a half and two-thirds of patients make a full and lasting recovery and many of the remainder are much improved.

A detailed manual describing CBT for bulimia nervosa has been available for many years - the latest version was published in 1993 (Fairburn et al, 1993b). The treatment has been adapted to suit patients with anorexia nervosa (Garner \& Bemis, 1985) but this adaptation has yet to be adequately evaluated. The role of CBT in the 